DOE/ER/40748-1

\title{
ADVANCED METHODS FOR THE COMPUTATION OF PARTICLE BEAM TRANSPORT AND THE COMPUTATION OF ELECTROMAGNETIC FIELDS AND BEAM-CAVITY INTERACTIONS
}

\author{
Progress Report
}

August 1992 - June 1993

\author{
Alex J. Dragt and Robert L. Gluckstern \\ Department of Physics \\ University of Maryland \\ College Park, Maryland 20742
}

June 1993

\section{PREPARED FOR THE U.S. DEPARTMENT OF ENERGY UNDER GRANT NUMBER DE-FG05-92ER40748}

\section{DISCLAIMER}

\footnotetext{
This report was prepared as an account of work sponsored by an agency of the United States Gerer employees, makes any warranty, express or implied, or assumes any legal liability or responsibility for the accuracy, completeness, or usefulness of any information, apparatus, product, or process disclosed, or represents that its use would not infringe privately owned rights. Reference herein to any specific commercial product, process, or service by trade name, trademark, manufacturer, or otherwise does not necessarily constitute or imply its endorsement, recommendation, or favoring by the United States Government or any agency thereof. The views and opinions of authors expressed herein do not necessarily state or reflect those of the United States Government or any agency thereof.
}

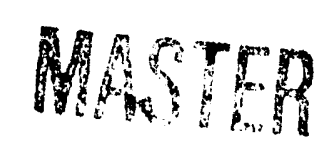




\section{INTRODUCTION}

The University of Maryland Dynamical Systems and Accelerator Theory Group has been carrying out long-term research work in the general area of Dynamical Systems with a particular emphasis on applications to Accelerator Physics. This work is broadly divided into two tasks: Charged Particle Beam Transport and the Computation of Electromagnetic Fields and BeamCavity Interactions. Each of these tasks is described briefly below. Work is devoted both to the development of new methods and the application of these methods to problems of current interest in accelerator physics including the theoretical performance of present and proposed high energy machines.

In addition to its research effort, the Dynamical Systems and Accelerator Theory Group is actively engaged in the education of students and postdoctoral research associates. To this end, it presents a regular graduate seminar course in accelerator physics, directs graduate students in M.S. and $\mathrm{Ph} . \mathrm{D}$. thesis research, and guides and fosters the research of Post-doctoral Research Associates, and Visiting Scientists.

Substantial progress in research has been made during the past year. There have also been important educational accomplishments. These achievements are summarized in the following report.

\subsection{Task A: Charged Particle Beam Transport}

A new method, employing Lie algebraic tools, has been developed for the computation of charged particle beam transport and accelerator design. It represents the action of each separate element of a beamline or accelerator, including nonlinear effects, by a certain operator. These operators can then be combined, following well-defined rules, to obtain a resultant transfer map that characterizes the entire system.

Lie algebraic methods may be used for particle tracking around or through a lattice and for analysis of linear and nonlinear lattice properties. When used for tracking, they are both exactly symplectic and extremely fast. Tracking can be performed element to element, lump to lump, or any mixture of the two. (A lump is a collection of elements combined together and treated by a single transfer map.) The speed for element to element tracking is comparable to that of other tracking methods. When collections of elements 
can be lumped together to form single transfer maps, tracking speeds can be orders of magnitude faster.

In addition to single particle tracking, Lie algebraic methods may also be used to determine how particle phase-space distribution functions evolve under transport through both linear and nonlinear elements. These methods are useful for the self consistent treatment of space-charge effects and for the study of how moments and emittances evolve. They also provide a means for characterizing beams in terms of invariant eigen emittances.

Lie algebraic methods also provide powerful means for carrying out analytic computations. Such computations include the calculation of first, second, and higher order chromaticities; first, second, and higher order dispersion; the dependence of tune on betatron amplitude; nonlinear lattice functions; nonlinear phase-space distortion; transfer map normal forms; nonlinear structure resonances; nonlinear beam-beam effects; and nonlinear invariants.

Finally, Lie algebraic methods can be used to give an explicit representation for the linear and nonlinear properties of the total transfer map of a system. This information can be used to evaluate or improve the optical quality of a single pass system such as a beam transport line or linear collider. For example, it is possible to design higher order achromats, and aberration corrected telescopes and final focus systems. For a circulating system such as a storage ring, explicit knowledge in this case of the one-turn transfer map can be used to predict the linear aperture of the system without the need for long-term tracking. It is hoped that eventually knowledge of this map can also be used to make predictions about the dynamic aperature of the system.

Presently the program MARY'LIE 3.0 implements these methods through third order. More advanced and higher order versions of MARYLIE are under development.

Lie algebraic methods are also applicable to many other areas including the design of high resolution electron microscopes and microprobes; the design of low energy wide acceptance spectrometers; light optics; and many other problems in the field of dy'namical sy'stems. Work is also being carried out in some of these areas. 


\subsection{Task B: Computation of Electromagnetic Fields and Beam-Cavity Interactions}

The next generation of linear colliders involves higher current beams of low emittance and short bunch length. The image currents produced by such beams generate wakefields which are capable of inducing instabilities in the beam. Accurate methods are needed to calculate these wakefields for iris loaded cavities and to analyze corrective measures intended to suppress these instabilities, such as BNS damping, autophasing, and detuning.

The next generation of high energy proton rings (LHC, SSC, Eloisatron) enters a parameter region where synchrotron radiation becomes increasingly important. Insertions into the beampipe are needed to remove the heat generated and to shield the beam pipe, usually operated at liquid helium temperatures, from the fields due to the circulating beam. The present plan is to insert a liner with holes into the beam pipe. Methods are needed to calculate the wakefields induced by such a liner, as well as the penetration of the fields due to the circulating beam into the region between the liner and the beam pipe.

The penetration of electromagnetic fields through small holes in a thick wall enters into a variety of problems of current interest. These include the coupling of wave guides to cavities, the construction of an accurate equivalent circuit for an iris loaded cavity, the design of beam position monitors, and the generation of wakefields by holes in a liner within a beam pipe. Methods are needed to extend the calculation, first done by Bethe in 1944 for circular and elliptical holes in a zero thickness wall for long wavelengths, to the case of thick walls and shorter wavelengths. 


\section{Educational Accomplishments}

One of the major goals of the Dynamical Systems and Accelerator Theory Group is the education of students, postdoctoral research associates, and visiting scientists. Listed below are present and past students associated with the program, postdoctoral research associates, and visiting scientists.

\subsection{Visiting Professor}

Dr. Iliya M. Kapchinsky was a Visiting Professor of Physics and Electrical Engineering from February 1, 1993 to April 30, 1993. During his visit he interacted with many colleagues and graduate students, who took advantage of his vast expertise in many aspects of accelerator theory. In addition, he presented about 10 lectures on the theory of linear accelerators. Unfortunately Dr. Kapchinsky died May 2, 1993, before he was able to return to Russia. His lectures notes are being assembled and will most likely be issued and circulated as a Los Alamos Report, since there is widespread interest in his perspectives and opinions on these subjects.

\subsection{Present Graduate Students}

Dan Abell

Thesis work in progress on the computation and analytic properties of transfer maps.

\section{Wen Hao Cheng}

Now in his third year as a Graduate Research Assistant. He has completed a study of an alternating phase focussing linac and is now working on the calculation of the transverse impedance of an obstacle in a beam pipe.

\section{Shicheng Jiang}

Now in his second year as a Graduate Research Assistant having completed work on the longitudinal impedance of a thick iris, and well on the way to completion of a corresponding calculation for the transverse impedance. 


\subsection{Past Graduate Students}

David Douglas, Ph.D. 1982.

Thesis: Lie Algebraic Methods for Particle Accelerator Theory.

Employment: Previously employed by the Lawrence Berkeley Laboratory. Presently employed by the Continuous Electron Beam Accelerator Facility.

Etienne Forest, Ph.D. 1984.

Thesis: Lie Algebraic Methods for Charged Particle Beams and Light Optics.

Employment: Lawrence Berkeley Laboratory.

Janko Milutinovic, Ph.D. 1984.

Thesis work done in Elementary Particle Theory while simultaneously receiving training in Accelerator Theory.

Employment: Accelerator Theory at Brookhaven National Laboratory.

Liam Healy, Ph.D. 1986.

Thesis: Lie Algebraic Methods for Treating Lattice Parameter Errors in Particle Accelerators.

Employment: Previously at CERN. Presently with celestial mechanics group at the Naval Research Laboratory.

Robert Ryne, Ph.D. 1987.

Thesis: Lie Algebraic Treatment of Space Charge.

Employment: Previously employed by Lawrence Livermore National Laboratory. Presently employed by the Los Alamos National Laboratory.

Petra Schuett (1987-88).

Worked one year at Maryland on Lie algebraic methods and Poisson solvers while simultaneously completing her Ph.D. thesis work on Wake-Field Acceleration under Thomas Weiland at DESY.

Employment: Theoretische Elektrotechnik Group at the Darmstadt Technische Hochschule. 
Govindan Rangarajan, Ph.D. 1990.

Thesis: Invariants for Symplectic Maps and Symplectic Completion of Symplectic Jets.

Employment: Previously at Lawrence Berkeley Laboratory. Now a member of the Mathematics Faculty, Indian Institute of Science, Bangalore India.

Rui Li, Ph.D. 1990.

Thesis: Analytic and Numerical Investigation of the Longitudinal Coupling Impedance

Employment: Continuous Electron Beam Accelerator Facility.

John Diamond, M.S. 1990.

Spent two years as a Graduate Research Assistant working on the penetration of electromagnetic fields through circular holes in a thick metallic wall. Also carried out symbolic manipulation program calculations for various complicated beamline elements such as combined function quadrupoles and combined function bends. In addition, responsible for general computational support. Now working at Adroit Systems, Alexandria, VA.

William Detlefs, M.S. (in Applied Math with Thesis) 1991.

Thesis: Longitudinal Coupling Impedance of a Thin Iris Collimator.

Employment: Now a graduate student with support in Physics at Cal Tech.

\subsection{Other Students}

Eric N. Opp (1984-85)

Spent one year working on the development of a computer program to calculate dispersions curves for azimuthally symmetric modes in an azimuthally symmetric periodic chain of coupled cavities. Now working in plasma physics.

Dimitris Vassiliadis (1987-88)

Spent $11 / 2$ years working as a Graduate Research Assistant on Alternating Phase Focussing. Now has received $\mathrm{Pl}$.D. in a group working on chaos. 
Jeffrey Skibo (1988-89)

Spent one year exploring possible further work on the use of Lie algebraic methods in the treatment of space charge. Also responsible for general computational support. Now has received Ph.D. in High Energy Astrophysics.

Eric Salter (1990-91)

Spent one year working on the calculation of the impedance of a thick iris. Also did programming for MARYLIE and provided general computational support. Now serving as a Teaching Assistant.

Glenn VanderWoude (1988-1992)

Undergraduate student and National Merit Scholar. Responsible for general computational support. Now holds a Graduate Research Assistantship in Applied Mathematics at the State University of New York (Stony Brook) where he is pursuing graduate work for the Ph.D.

\subsection{Present Ph.D. Professional Staff}

Ivan Gjaja (1991-)

Postdoctoral Research Associate and SSC Fellow.

Hiromi Okamoto (1992-)

Visiting Scientist. Partially supported by Japanese Government.

Huanchun Ye (1993-)

Postdoctoral Research Associate.

\subsection{Past Ph.D. Professional Staff}

Herbert O. Moser (1985)

Visiting Scientist. Presently at IMM Institut fur Mikrotechnik, Mainz Germany. 
Hiromi Okamoto (1989-90)

Visiting Scientist. Permanently at Nuclear Science Laboratory, Institute for Chemical Research, Kyoto Japan.

Filippo Neri (1983-90)

Postdoctoral Research Associate. Presently at Los Alamos National Laboratory.

Fernando Casas (1992-1993)

Visiting Postdoctoral Fellow, supported by Spanish Government.

Branislav Radak (1992-1993)

Postdoctoral Research Associate.

Johannes van Zeijts (1987-1993)

Postdoctoral Research Associate. Presently at Continuous Electron Beam Accelerator Facility. 


\section{Research Accomplishments}

Several of the objectives set forth in the 1992 proposal have been met, and additional objectives have been achieved as well. A brief summary of work accomplished over the last year is given below.

\subsection{Task A: Charged Particle Beam Transport}

1. The program MARYLIE 3.0, comprising approximately 32,000 lines of code, is nearly ready for wide distribution and general use. $A$ detailed instruction manual, consisting of some 470 pages and set in TEX, is substantially complete. ${ }^{1}$ Preliminary versions of the code have been tested on VAX, CRAY, IBM, SUN, and UNIX computers, and are in use in various laboratories in the United States, Canada, and Europe. The use of MARYLIE will be taught as part of the subject matter in the Summer 1993 U.S. Particle Accelerator School.

2. Continued improvements are being made in MARYLIE 3.0. Work is currently being done on optimizing routines. These routines adjust parameters to optimize arbitrary merit functions, and are of use when fits are not possible.

3. A version of MARYLIE 3.1, a third-order code including error effects, has been assembled. The computation of error effects in nonlinear Hamiltonian systems using Lie algebraic methods has been worked out in detail, and applied to the problem of steering magnets and mispowered dipoles. ${ }^{2}$ These computations are being implemented in MARYLIE 3.1. It is intended to use this version of MARYLIE to make preliminary studies of the SSC.

4. Programming has begun on MARYLIE 5.1, a fifth-order code inciuding error effects. Recent work shows that long-term tracking studies for the SSC can be made using a fourth-order full one-turn map and a generating function symplectification scheme. ${ }^{3,4}$ This method is orders of magnitude faster than element by element tracking, and will pernit much more detailed study of the anticipated performance of the SSC. All aspects of this method (production and use of a one-turn map and 
generating; function symplectification) were originally conceived and developed at Maryland. ${ }^{5,6,7}$ MARYLIE 5.1 already implements these methods fully and should be an ideal code for SSC studies. Work on the use of MARYLIE 3.1 (see item 3 above) and MARYLIE 5.1 for SSC studies is being carried out in collaboration with Thomas Mottershead and Yiton Yan at the SSC.

5. A new hybrid code that uses both Taylor series and Lie algebraic methods has been developed. This code, called Tlie, works numerically to arbitrary order. ${ }^{8}$ That is, given any Hamiltonian expanded to some order, it can compute by numerical means the associated transfer map to the same order. It can also multiply maps of any specified order. Tlie has been used to cross check other existing codes, and for fifth and higher order aberration studies at Los Alamos. In addition, it has been used to study the importance of misalignment and higher-order aberration effects for the Stanford Final Focus Test Beam Facility. ${ }^{9}$ Collaborative work, with J. van Zeijts and D. Douglas at CEBAF, has begun to increase the capacities of Tlie so that it can handle charged particle beam transport with acceleration.

6. The use of Taylor series maps to describe charged particle beam transport raises at least two questions. First, what is the domain of convergence of the series? Second, how can a truncated expansion be made symplectic? Work has begun on the first question as part of the thesis research of Dan Abell, and results have been found for the anharmonic oscillator. ${ }^{10}$ This work requires extensive use of the theory of several complex variables and leads to remarkable results. For example, the Hamiltonians $H=\left(p^{2}+q^{2}\right) / 2 \pm q^{4}$ both lead to Taylor maps having identical convergence domains.

7. Work on the second question in item 6 above began with the use of generating functions. ${ }^{5,6,7}$ Subsequently Irwin proposed a method that led to Cremona maps. ${ }^{711}$ Dragt, Rangarajan, Gjaja, and Abell continue to work on Cremona map and solvable map approaches. ${ }^{12,13,14,15,16}$ One of these solvable map approaches, that of monornial factorization, has been implemented in MARYLIE 3.0. Some of this work was described in an invited talk presented at the 1992 Brookhaven Workshop on the 
Stability of Particle Motion in Storage Rings, and some is expected to be part of the thesis research of Dan Abell.

8. Work has continued on writing an extensive set of Lecture Notes on Nonlinear Dynamics and Lie Methods with Applications to Accelerator Physics. ${ }^{17}$

9. In collaboration with others a lengthy publication has been prepared describing the modern map approach to charged particle beam dynamics. ${ }^{18}$

10. Sufficient conditions have been derived for the convergence of the infinite product of Lie transformations that arises in the description of an arbitrary analytic nonlinear map. ${ }^{19}$ Work is in progress on relating this result to the conditions for convergence of Taylor series maps.

11. A Lie algebraic formulation of light ray optics has been developed earlier. ${ }^{20,21}$ This work has now been extended to the case of light wave optics including interference effects. A lengthy publication on this subject is in preparation. ${ }^{22}$

12. A Lie algebraic description of charged particle beam transport including space charge effects has been developed earlier. ${ }^{23,24}$ At that time it was difficult to make comparisons between Lie algebraic methods and traditional particle in celi methods due to cell particle population fluctuations. With the advent of massively parallel computers such as the CM5, it is now possible to run particle in cell codes with a very large number of particles, and thereby reduce particle population fluctions within individual cells. In collaboration with Robert Ryne at Los Alamos, work has resumed on comparing Lie algebraic and particle in cell results. 


\subsection{Task B: Computation of Electromagnetic Fields and Beam-Cavity Interactions}

1. Alternating Phase Focussing (APF)

The difference equations for particle motion in an alternating phase focussing linac have been used to calculate the separatrix to be expected neglecting space charge, and from that, to estimate the current carrying capacity of such a structure [a]. These predictions were compared with subsequent numerical orbit calculations [b] using PARMILA, which includes space charge, and it was found that significant emittance growth took place. Based on the predictions [c] that the parametric resonance between the longitudinal and transverse motion would be important for symmetric APF, we analyzed this resonance, finding two approximate constants of the motion, and predicting those parameter regions where emittance growth would or would not take place, confirming the PARMILA results [d].

2. Coupling Impedance of a Thick Iris

Equations were derived for calculation of the longitudinal impedance of an iris in a beam pipe. These were implemented in a computer program to calculate the impedance [e], and limiting results were obtained for a large beam pipe radius, corresponding to the configuration of a hole in a thick wall plate.

Subsequent work focussed on the transverse impedance and results are now being obtained for a wide variation of parameters, including the configuration of a hole in a thick wall plate [f]. We ultimately hope to address the corresponding situation of a periodic array of plates or a periodic iris loaded cavity.

3. Penetration of Fields through Holes in a Conducting Wall

We have started to analyze the penetration of fields through holes of elliptical and rectangular cross section. In addition we are constructing variational approaches to calculating the effect of a circular hole in those cases where the hole radius and wall thickeness are comparable with the wave length. Knowledge of such frequency dependent corrections for the polarizability and susceptibility of the hole are important in many examples. 


\section{Holes in a Liner}

Work has been completed on the impedance of a hole in a beam pipe [g] and on the impedance of many holes in a liner within a beam pipe [h], a configuration under design at both the LHC and the SSC to remove synchrotron radiation from the protons. In the course of this work, we also were able to obtain the resistive wall impedance in both beam pipes of elliptical and rectangular cross section [i].

5. Free Electron Lasers (FEL)

We have successfully analyzed the saturation region for an FEL in the Compton regime, and in particular, how the transition from the exponential region to the bunched beam in the saturation region takes place [j]. This one dimensional model is now being extended to include a finite radius electron beam. We then plan to apply this analysis to a tapered FEL.

6. Wakefields in Linear Colliders

Work has been completed on the development of an equivalent circuit model that accurately describes the evolution of wakefields in a detuned iris loaded wave guide $[k, \ell]$. This work is now being extended to include the further damping of the deflecting modes through a lossy manifold. 


\section{REFERENCES}

\subsection{Task A: Charged Particle Beam Transport}

1. A.J. Dragt et al., MARYLIE 3.0 User's Manual, A Program for Charged Particle Beam Transport Based on Lie Algebraic Methods (1993).

2. L. Healy, A.J. Dragt, and I. Gjaja, Computation of Error Effects in Nonlinear Hamiltonian Systems Using Lie Algebraic Methods, Journal of Mathematical Physics, 33, p. 1948 (1992).

3. Y.T. Yan, A.J. Dragt, et al., Comment on Round-Off Errors and on One-Turn Taylor Maps, Nonlinear Problems in Future Particle Accelerators, W. Scandale and G. Turchetti, Ed., World Scientific (1991).

4. Y.T. Yan, P.J. Channell, and M.J. Syphers, Peformance of an Algorithm for Symplectic Implicit One-Turn-Map Tracking, SSCL-Print-157 (1993).

5. D.R. Douglas and A.J. Dragt, Lie Algebraic Methods for Particle Tracking Calculations, Proceedings of the 12th International Conference on High Energy Accelerators, F.T. Cole and R. Donaldson eds. (Fermilab 1983).

6. D.R. Douglas, E. Forest, and R. Servranckx, A Method to Render Second Order Beam Optics Program Symplectic, IEEE Tran. on Nucl. Sci. NS-325, 2279 (19S5).

7. A.J. Dragt et al., Lie Algebraic Treatment of Linear and Nonlinear Beam Dynamics, Annual Review of Nuclear and Particle Science, vol. 38, p. 455 (1988).

8. J. van Zeijts and F. Neri, The Arbitrary Order Design Code Tlie 1.0 (1992).

9. A.J. Dragt, Review of the SLAC Final Focus Test Beam Facility (1990).

10. A.J. Dragt and D.T. Abell, Taylor Series Maps and Their Domain of Convergence, Proceedings of the 1992 Brookhaven Workshop on the 
Stability of Particle Motion in Storage Rings (to be published by American Institute of Physics in 1993).

11. J. Irwin, A Multi-kick Factorization Algorithm for Nonlinear Maps, SSCL-228 (1989).

12. A.J. Dragt, I.M. Gjaja, and G. Rangarajan, Kick Factorization of Symplectic Maps, Proceedings of the IEEE Accelerator Conference, p. 1621 (1991).

13. G. Rangarajan, Invariants for Symplectic Maps and Symplectic Completion of Symplectic Jets, Ph.D. thesis, Physics Department, University of Maryıand (1990).

14. A.J. Dragt and D.T. Abell, Jolt Factorization of Symplectic Maps, Int. J. Mod. Phys. A (Proc. Suppl.) 2B, p. 1019, World Scientific (1993).

15. I. Gjaja, A.J. Dragt, and D.T. Abell, A Comparison of Methods for Long-Term Tracking Using Symplectic Maps, Proceedings of the Berlin Workshop on Nonlinear Problems in Accelerator Physics (to be published by Adam Hilger in 1993).

16. I. Gjaja, Monomial Factorization of Symplectic Maps, submitted to Particle Accelerators (1993).

17. A.J. Dragt, Lectures on Nonlinear Dynamics and Lie Methods with Applications to Accelerator Physics (225 pp.)(1992).

18. E. Forest, L. Michelotti, A.J. Dragt, and S.J. Berg, The Moden Approach to Single Particle Dynamics for Circular Rings, Proceedings of the 1992 Brookhaven Workshop on the Stability of Particle Motion in Storage Rings (to be published by American Institute of Physics in 1993).

19. I. Gjaja, Convergence of an Infinite Product of Lie Transformations, manuscript in preparation (1993).

20. A.J. Dragt, A Lie Algebraic Theory of Geometrical Optics and Optical Aberrations, J. Opt. Sci. Am. 72, p. 372 (1982). 
21. A.J. Dragt, E. Forest, and K. Wolf, Foundations of a Lie Algebraic Theory of Geometrical Optics, published in Lie Methods in Optics, J.S. Mondragon and K.B. Wolf, editors, Springer-Verlag (1986).

22. A.J. Dragt, Lie Algebraic Methods for Ray and Wave Optics, manuscript in preparation (1993).

23. R.D. Ryne, Lie Algebraic Treatment of Space Charge, Ph.D. thesis, Department of Physics, University of Maryland (1987).

24. R. Ryne and A.J. Dragt, Magnetic Optics Calculations for Cylindrically Symmetric Beams with Space Charge, Particle Accelerators 35, p. 129 (1991). 


\subsection{Task B: Computation of Electromagnetic Fields and Beam-Cavity Interactions}

[a] R.L. Gluckstern and D. Vassiliadis, Low Beta Linacs and Alternating Phase Focusing, Proc. of the Linac Conference, Williamsburg, VA, Oct. 1988 , p. 112.

[b] R.L. Gluckstern, W-H. Cheng, S. Nath and T.P. Wangler, Alternating Phase Focusing Including Space Charge, Proceedings of the Linac Conference, Ottawa, August 1992, p. 193.

[c] H. Okamoto, Coupled Motion in Alternating Phase Focused Linacs, Bull. Inst. for Chem. Res., Kyoto Univ., 9, No. 1 (1991).

[d] W-H Cheng, R.L. Gluckstern, and H. Okamoto, Synchrobetatron Resonance Effects in Alternating Phase Focusing, Proceedings of the Particle Accelerator Conference, Washington, DC, May 1993.

[e] R.L. Gluckstern and S. Jiang, Longitudinal Coupling Impedance of a Thick Iris Collimator, Proceedings of the Linac Conference, Ottawa, August 1992, p. 477.

[f] S. Jiang, H. Okamoto, and R.L. Gluckstern, Transverse Impedance of an Iris in a Beam Pipe, Proceedings of the Particle Accelerator Conference, Washington, DC, May 1993.

[g] R.L. Gluckstern, Coupling Impedance of a Single Hole in a Thick Wall Beam Pipe, Phys. Rev. A15, $\underline{46}, 1106$ (1992).

[h] R.L. Gluckstern, Coupling Impedance of Many Holes in a Liner Within a Beam Pipe, Phys. Rev. A15, 트, 1110 (1992).

[i] R.L. Gluckstern, J. van Zeijts and B. Zotter, Coupling Impedance of Beam Pipes of General Cross Section, Phys. Rev. E, 4ㄱ, 656 (1993). 
[j] R.L. Gluckstern, S. Krinsky and H. Okamoto, An Analysis of the Saturation of a High Gain FEL, to be published in Physical Review E (1993).

[k] K.L.F. Bane and R.L. Gluckstern, The Transverse Wakefield of a Detuned $\mathrm{X}$-Band Accelerator Structure, to be published in Particle Accelerators, 1993.

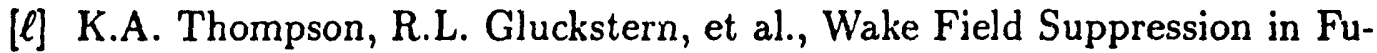
ture Linear Colliders, submitted to Particle Accelerators (1992). 


\section{Publications and Reports Written During Grant Period}

\subsection{Task A}

A.J. Dragt and D.T. Abell, Jolt Factorization of Symplectic Maps, Int. J. Mod. Phys. A (Proc. Suppl.) 2B, p. 1019, World Scientific (1993).

I. Gjaja, A.J. Dragt, and D.T. Abell, A Comparison of Methods for LongTerm Tracking Using Symplectic Maps, Proceedings of the Berlin Workshop on Nonlinear Problems in Accelerator Physics (to be published by Adam Hilger in 1993).

I. Gjaja, Monomial Factorization of Symplectic Maps, submitted to Particle Accelerators (1993).

I. Gjaja, Convergence of an Infinite Product of Lie Transformations, manuscript in preparation (1993).

E. Forest, L. Michelotti, A.J. Dragt, and S.J. Berg, The Moden Approach to Single Particle Dynamics for Circular Rings, Proceedings of the 1992 Brookhaven Workshop on the Stability of Particle Motion in Storage Rings (to be published by American Institute of Physics in 1993).

A.J. Dragt and D.T. Abell, Taylor Series Maps and Their Domain of Convergence, Proceedings of the 1992 Brookhaven Workshop on the Stability of Particle Motion in Storage Rings (to be published by American Institute of Physics in 1993).

A.J. Dragt and F. Neri, Chromaticity of the Los Alamos Proton Storage Ring, LANL Technical Report AT3:TN-92-12. 


\subsection{Task B}

R.L. Gluckstern, Coupling Impedance of a Single Hole in a Thick Wall Beam Pipe, Phys. Rev. A15, 6ㅜ, 1106 (1992).

R.L. Gluckstern, Coupling Impedance of Many Holes in a Liner Within a Beam Pipe, Phys. Rev. A15, $\underline{46}, 1110$ (1992).

R.L. Gluckstern and F. Ruggiero, Variational Formulation for Linear Optics in a Periodic Focussing System, Particle Accelerators, 모, 125 (1992).

K.L.F. Bane and R.L. Gluckstern, The Transverse Wakefield of a Detuned X-Band Accelerator Structure, to be published in Particle Accelerators, 1993.

K.A. Thompson, R.L. Gluckstern, et al., Wake Field Suppression in Future Linear Colliders, submitted to Particle Accelerators (1992).

R.L. Gluckstern, J. van Zeijts, and B. Zotter, Coupling Impedance of Beam Pipes of General Cross Section, Phys. Rev. E, 47, 656 (1993).

R.L. Gluckstern, S. Krinsky, and H. Okamoto, An Analysis of the Saturation of a High Gain FEL, to be published in Physical Review E (1993).

R.L. Gluckstern and S. Jiang, Longitudinal Coupling Impedance of a Thick Iris Collimator, Proceedings of the Linac Conference, Ottawa, August 1992, p. 477.

R.L. Gluckstern, J. van Zeijts, and B. Zotter, Coupling Impedance in an Elliptical Beam Pipe, Proceedings of the Linac Conference, Ottawa, August 1992 , p. 480.

R.L. Gluckstern, W-H. Cheng, S. Nath, and T.P. Wangler, Alternating Phase Focussing Including Space Charge, Proceedings of the Linac Conference, Ottawa, August 1992, p. 193. 
R.L. Gluckstern and R. Li, Cavity Height Dependence of the Longitudinal Impedance for a Pillbox Cavity at High Frequency, Proceedings of the Linac Conference, Ottawa, August 1992 p. 755.

1. Gjaja and R.L. Gluckstern, Longitudinal Coupling Impedance of a Cavity, Proceedings of the Particle Accelerator Conference, Washington, DC, May 1993.

W-H Cheng, R.L. Gluckstern, and H. Okamoto, Synchrobetatron Resonance Effects in Alternating Phase Focusing, Proceedings of the Particle Accelerator Conference, Washington, DC, May 1993.

R.L. Gluckstern,S. Krinsky, and H. Okamoto, Saturation of a High Gain FEL, Proceedings of the Particle Accelerator Conference, Washington, DC, May 1993.

S. Jiang, H. Okamoto, and R.L. Gluckstern, Transverse Impedance of an Iris in a Beam Pipe, Proceedings of the Particle Accelerator Conference, Washington, DC, May 1993.

R.L. Gluckstern, Methods of Impedance Calculation (Invited Paper), Proceedings of the Particle Accelerator Conference, Washington. DC, May 1993.

R.L. Gluckstern, J. van Zeijts, and B. Zotter, Coupling Impedance of a Hole in an Elliptical Beam Pipe, CERN Report SL/92-18 (AP) (1992).

R.L. Gluckstern, Reflection and Transmission Coefficients of a Hole or Slot in a Thick Wall Separating Two Coaxial Guides, CERN Report SL/Notes92-31 (AP) (1992). 


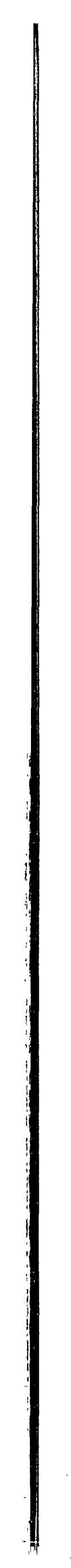

